\title{
Descripción de hallazgos radiológicos en psitácidos decomisados y mantenidos en un centro de rescate en Tingo María, Huánuco-Perú
}

\author{
Description of radiological findings in psitacids seized and kept in a rescue center \\ in Tingo María, Huánuco - Peru
}

\author{
Ricardo Grandez R. ${ }^{1,2,4}$, Catalina Hermoza G. ${ }^{3}$, Sheilla Tirmizi B. ${ }^{1}$
}

\section{Resumen}

\begin{abstract}
El tráfico ilegal de aves, especialmente de psitácidas es frecuente en Perú. Los métodos de captura y las malas condiciones de transporte y cautividad hacen que sufran traumas y lesiones físicas, que llegan a dañar estructuras óseas. Estos animales son decomisados y enviados a centros de rescate con la finalidad es rehabilitarlos para su reintroducción. En el presente estudió se evaluaron los registros radiográficos de nueve psitácidos de un centro de rescate en Tingo María, Perú (Ara chloropterus, Amazona farinosa, Ara macao y Pionus menstruus [uno por especie], dos Ara militatis y tres Ara ararauna). La mayoría de las lesiones ocurrieron en el miembro anterior (77\%), sobre todo en la porción distal (44\%); siendo el radio y la ulna los más afectados. Las complicaciones más comunes fueron callo óseo exuberante, mala consolidación y osteomielitis; y las secuelas clínicas fueron pérdida la capacidad de vuelo y claudicación marcada. Las lesiones halladas impiden la reintroducción de estas aves a su hábitat natural.
\end{abstract}

Palabras clave: psitácidos, radiología, fracturas

${ }^{1}$ Sección de Biociencias y Ciencias Clínicas, Departamento Académico de Medicina Veterinaria y Zootecnia, Facultad de Medicina Veterinaria y Zootecnia, Universidad Peruana Cayetano Heredia, Lima, Perú

${ }^{2}$ Laboratorio de Vida Silvestre, Facultad de Medicina Veterinaria y Zootecnia, Universidad Peruana Cayetano Heredia, Lima, Perú

${ }^{3}$ Centro de Rescate Mundo Natural, Tingo María, Huánuco, Perú

${ }^{4}$ E-mail: rgrandez@hotmail.com

Recibido: 21 de julio de 2020

Aceptado para publicación: 16 de enero de 2021

Publicado: 24 de abril de 2021 
Illegal trafficking in birds, especially parrots, is frequent in Peru. The methods of capture and the poor conditions of transport and captivity cause them to suffer trauma and physical injuries, which can damage bone structures. These animals are confiscated and sent to rescue centres to rehabilitate them for their reintroduction. In the present study, the radiographic records of nine parrots from a rescue centre in Tingo María, Peru (Ara chloropterus, Amazona farinosa, Ara macao and Pionus menstruus [one per species], two Ara militatis, and three Ara ararauna) were evaluated. Most of the injuries occurred in the forelimb (77\%), especially in the distal portion (44\%); the radius and ulna being the most affected. The most common complications were exuberant bony callus, poor consolidation and osteomyelitis; and the clinical sequelae were loss of flight capacity and marked claudication. The lesions found prevent the reintroduction of these birds to their natural habitat.

Key words: psittacines, radiology, fractures

\section{INTRODUCCIÓN}

El tráfico de vida silvestre está dentro de los comercios ilícitos más lucrativos del mundo e incluye la captura, caza furtiva y contrabando de especímenes y sus derivados o productos (South y Wyatt, 2011). El Perú es uno de los países con mayor cantidad de tráfico ilegal de especies amazónicas. Según el Servicio Nacional Forestal y de Fauna Silvestre (SERFOR), las aves son la clase de vertebrados más traficadas, especialmente las psitácidas (SERFOR, 2017), lo cual aunado a la pérdida y degradación de su hábitat ha hecho que sean uno de los grupos de aves más amenazados.

Las condiciones en las que se capturan, transportan y comercializan los animales silvestres son las causas principales de lesiones y muerte de estos, por lo que se estima que los volúmenes extraídos son mucho mayores a los reportados. Aquellos animales que sobreviven son sujetos a técnicas de manejo que no alcanzan estándares mínimos de bioseguridad y bienestar animal. Por lo tanto, cuando son rescatados y enviados a centros autorizados por SERFOR, deben pasar por un examen veterinario para determinar su estado de salud y otorgarles el tratamiento necesario a sus lesiones (WCS y SERFOR, 2016). Estos exámenes deberían incluir pruebas radiológicas en animales con sospecha de fracturas; sin embargo, muchas veces este procedimiento no se puede realizar.

Los huesos de las aves son más ricos en sustancias inorgánicas que los de los mamíferos, llegando a contener hasta un $84 \%$ de estas sustancias (Doneley, 2016). Los huesos largos presentan una cortical muy fina y frágil; y la cavidad medular ancha que contiene una red de trabéculas que aumentan la resistencia del hueso; asimismo, algunos huesos pueden ser neumáticos. Estas circunstancias dan lugar a que los huesos de las aves sean más duros, pero a la vez más frágiles y menos elásticos que los de los mamíferos; siendo por lo tanto más susceptibles a sufrir fracturas (Doneley, 2016; McRee et al., 2017).

La radiología es un método de diagnóstico complementario para la evaluación de diversos sistemas, especialmente el esquelético (Doneley, 2016); permitiendo diagnosticar, evaluar y tomar decisiones médico-qui- 
rúrgicas de traumas, fracturas, luxaciones, anomalías del desarrollo, neoplasias óseas y cuerpos extraños, como balas o perdigones (Beregi et al., 1999). El esqueleto del ave, como en todo vertebrado, se divide en axial y apendicular, El axial esta conformado por el esqueleto cefálico y el raquis, que se divide en sus porciones cervical, torácica, lumbosacra y coccígea. El esqueleto apendicular esta conformado por el miembro anterior, torácico o ala; y por el miembro posterior, pélvico o pata (Cano, 2010; Silverman y Tell, 2010; Kilbourne, 2013; Chin et al., 2017)

Cada interpretación radiográfica debería incluir imágenes tomadas en al menos dos proyecciones, laterales y ventro-dorsales. El posicionamiento correcto es imprescindible para que los resultados radiográficos se evalúen adecuadamente (KrautwaldJunghanns y Schmidt, 2012); considerándose que los estudios de las extremidades o miembros torácicas consideren las proyecciones mediolateral y caudocranial. Se debe considerar que las técnicas de todo el cuerpo dan lugar a proyecciones ortogonales de las extremidades o miembros pélvicos, por lo que no se requieren técnicas de posicionamiento adicionales (Silverman y Tell, 2010).

Las fracturas en las aves con frecuencia son abiertas y conminutas debido a la cobertura mínima de los tejidos blandos que posee (Doneley, 2016); siendo el ala la parte más susceptible a las lesiones, que a menudo resultan fatales debido a las repercusiones negativas que producen sobre el vuelo. Las fracturas articulares se encuentran entre las más devastadoras de todas las lesiones de las alas, pues generan una discapacidad para el vuelo que hace difícil o imposible que las aves se alimenten adecuadamente (Bennett et al., 1997). Entre las principales complicaciones de las fracturas descritas en aves están la pseudoartrosis, la osteomielitis y la osteoartritis (Arnaut, 2006, Farrow, 2008; Doneley, 2016).
El propósito del presente estudio fue describir y caracterizar los hallazgos radiológicos sobre complicaciones de lesiones y secuelas clínicas de fracturas originados en psitácidos decomisados y mantenidos en un centro de rescate en Tingo María, Perú.

\section{Materiales y Métodos}

\section{Lugar y Periodo}

El estudio fue realizado en el centro de rescate «Mundo Natural», ubicado en la ciudad de Tingo María, provincia Leoncio Prado, departamento de Huánuco, Perú. Las radiografías fueron tomadas por el médico veterinario a cargo del centro, como parte de los controles sanitarios de recepción del animal y control sanitario de rutina. Las tomas realizadas corresponden al periodo de julio de 2016 a mayo de 2018 .

El trabajo comprendió el estudio radiográfico de los siguientes especímenes: Ara chloropterus, Amazona farinosa, Ara macao y Pionus menstruus (uno por especie), dos Ara militatis y tres individuos de la especie Ara ararauna. Los nueve individuos se encontraban en edad adulta.

\section{Procesamiento Radiográfico}

La contención y sedación fue realizada por el médico veterinario del centro de rescate. Los animales fueron pesados y dosificados vía IM con midazolam a dosis de $2 \mathrm{mg} / \mathrm{kg}$ (Hawkins et al., 2013), obteniéndose una sedación completa por 20 minutos. Para las tomas radiográficas se utilizó el equipo de Rayos X MinXray HF 100 perteneciente al Zoológico Granja Villa y su Mundo Mágico. Se emplearon placas radiográficas Kodak Medical X-Ray Film General Purpose Green de 27.9 x $35.6 \mathrm{~cm}$. Los valores de exposición empleados fueron de $20 \mathrm{~mA}, 65 \mathrm{kV}$ y con tiempo de exposición de $0.045 \mathrm{~s}$. 
Se obtuvieron placas en posición ventro dorsal o lateral del cuerpo completo de cada ave. Las placas fueron reveladas manualmente utilizando los líquidos de revelado de Kodak GBX Developer and Replenisher por $30 \mathrm{~s}$ y de fijación de Kodak GBX Fixer and Replenisher por $2 \mathrm{~min}$.

Las placas fueron revisadas con el uso del negatoscopio convencional. Se tomaron registros fotográficos de todas las radiografías para identificar y describir las lesiones, en tanto que la observación y el examen clínico permitió determinar las secuelas presentes. Las lesiones en huesos y articulaciones afectadas se determinaron y clasificaron según la región anatómica involucrada:

- Miembro anterior o torácico: ala proximal: articulación humeral (hombro) y húmero; ala distal: articulación cubital (codo), radio, ulna, articulación radio carpiana (muñeca), hueso carporadial, hueso cubital del carpo, metacarpiano mayor y menor, álula, falanges del digital menor y falanges del digital mayor.

- Miembro posterior o pélvico: pata proximal: articulación coxo-femoral y fémur; pata distal: articulación femorotibiotarsiana (rodilla), tibiotarso, fíbula, tarsometatarso, falanges y digitales I, II, III y IV.

Las complicaciones en la consolidación se clasificaron en: formación callosa exuberante, lesión sin consolidación, mala consolidación, osteomielitis, artrosis secundaria e inflamación del tejido blando. Asimismo, las secuelas clínicas causadas por las lesiones se clasificaron en: acortamiento del miembro afectado, desviación del eje del miembro, pérdida de la movilidad articular, claudicación y pérdida de la capacidad de vuelo. Los datos se presentan mediante estadística descriptiva.

\section{Resultados}

Se evaluaron 25 radiografías de los nueve psitácidos considerados en el estudio. El resumen de las lesiones, complicaciones y secuelas halladas en cada espécimen se muestran en los cuadros 1 y 2 . Los resultados de las lesiones según región anatómica se muestran en el Cuadro 3, donde se evidencia que el $77 \%$ de las lesiones se encontraron en los miembros torácicos, siendo más afectada la porción distal ( $44 \%$ del total de las lesiones). Tanto el radio y la ulna fueron los huesos más afectados y en la misma proporción.

Las complicaciones de las lesiones más comunes fueron la presencia de callo óseo exuberante, mala consolidación y osteomielitis. Tanto el callo óseo exuberante como la mala consolidación se presentaron en todas las lesiones de fracturas antiguas (Cuadro 4), en tanto que, con relación a las secuelas clínicas, todas las aves cuyas lesiones fueron a nivel del ala perdieron la capacidad de vuelo, y en aquellas con lesiones a nivel de miembros posteriores o pélvicos presentaban claudicación marcada. Varias aves perdieron la movilidad articular de las articulaciones afectadas (Cuadro 5). Las imágenes radiográficas más representativas de las lesiones en extremidades se muestran en las figuras 1 y 2 .

\section{Discusión}

Las lesiones observadas en aves asociadas al tráfico ilegal pudieron ocurrir durante su captura y transporte. Según WCS y SERFOR (2016), los psitácidos anidan en las cavidades de los troncos de los árboles, y son cazados mediante la tala de estos; lo que ocasiona golpes y caídas que pueden terminar en fracturas óseas. Las aves son usualmente 
Cuadro 1. Hallazgos radiográficos en nueve psitácidos rescatados de tráfico ilegal de especies silvestres y mantenidos en cautiverio en un centro de rescate en Tingo María, Huánuco, Perú (2016-2018) - Parte I

\begin{tabular}{|c|c|c|c|}
\hline $\begin{array}{l}\text { Especie / } \\
\text { extremidad } \\
\text { afectada }\end{array}$ & $\begin{array}{l}\text { Huesos / } \\
\text { articulación } \\
\text { afectada }\end{array}$ & Descripción de la lesión & Secuela clínica \\
\hline $\begin{array}{l}\text { Ara } \\
\text { chloropterus } \\
\text { / anterior } \\
\text { derecha }\end{array}$ & $\begin{array}{l}\text { Huesos: } \\
\text { radio, ulna, } \\
\text { carporadial, } \\
\text { cubital del } \\
\text { carpo, } \\
\text { metacarpiano } \\
\text { mayor y } \\
\text { metacarpiano } \\
\text { menor. } \\
\text { Articulación: } \\
\text { muñeca }\end{array}$ & $\begin{array}{l}\text { Fracturas completas de los huesos } \\
\text { carporadial, cubital del carpo, diáfisis } \\
\text { distal de radio y ulna, diáfisis } \\
\text { proximal del metacarpiano mayor y } \\
\text { menor; afección de la articulación } \\
\text { radiocarpiana. Se observa } \\
\text { osteomielitis y mala consolidación } \\
\text { que ha generado un callo exuberante / } \\
\text { artrosis secundaria. }\end{array}$ & $\begin{array}{l}\text { Pérdida de la } \\
\text { movilidad de la } \\
\text { articulación y pérdida } \\
\text { de la capacidad de } \\
\text { vuelo. }\end{array}$ \\
\hline $\begin{array}{l}\text { Ara militatis } \\
1 \\
\text { / anterior } \\
\text { izquierda }\end{array}$ & $\begin{array}{l}\text { Huesos: radio } \\
\text { y ulna }\end{array}$ & $\begin{array}{l}\text { Fracturas simples y completas del } \\
\text { radio y ulna. En la ulna se observa } \\
\text { una secuela de fractura transversal y } \\
\text { en el radio una secuela de fractura } \\
\text { oblicua con desplazamiento lateral. } \\
\text { En ambos se observa una mala } \\
\text { consolidación y callos exuberantes. }\end{array}$ & $\begin{array}{l}\text { Acortamiento discreto } \\
\text { del miembro. Pérdida } \\
\text { de la capacidad de } \\
\text { vuelo. }\end{array}$ \\
\hline $\begin{array}{l}\text { Ara militatis } \\
2 \\
\text { / anterior } \\
\text { derecha }\end{array}$ & $\begin{array}{l}\text { Huesos: } \\
\text { húmero } \\
\text { Articulación: } \\
\text { hombro }\end{array}$ & $\begin{array}{l}\text { Fractura múltiple, completa y } \\
\text { conminuta del húmero (en cabeza, } \\
\text { tuberosidades y cuerpo). Se observa } \\
\text { osteólisis y osteomielitis afectando } \\
\text { articulación humeral, artrosis, mala } \\
\text { consolidación y formación de callo } \\
\text { exuberante. }\end{array}$ & $\begin{array}{l}\text { Acortamiento del } \\
\text { miembro, pérdida de } \\
\text { movilidad articular y } \\
\text { pérdida de la } \\
\text { capacidad de vuelo. }\end{array}$ \\
\hline $\begin{array}{l}\text { Ara ararauna } \\
1 \\
\text { / anterior } \\
\text { derecha }\end{array}$ & $\begin{array}{l}\text { Huesos: } \\
\text { coracoides } \\
\text { Articulación: } \\
\text { hombro }\end{array}$ & $\begin{array}{l}\text { Presencia de osteomielitis con } \\
\text { osteólisis del hueso coracoides } \\
\text { derecho. Se observa formación de } \\
\text { callo exuberante y pérdida de } \\
\text { articulación humeral. }\end{array}$ & $\begin{array}{l}\text { Pérdida de la } \\
\text { movilidad de la } \\
\text { articulación del } \\
\text { hombro y la capacidad } \\
\text { de vuelo. }\end{array}$ \\
\hline $\begin{array}{l}\text { Ara ararauna } \\
2 \\
\text { / anterior } \\
\text { derecha }\end{array}$ & $\begin{array}{l}\text { Huesos: radio } \\
\text { y ulna }\end{array}$ & $\begin{array}{l}\text { Fracturas diafisarias simples de ulna } \\
\text { y radio. En ambos huesos se ha } \\
\text { producido una mala consolidación y } \\
\text { callo exuberante. }\end{array}$ & $\begin{array}{l}\text { Acortamiento y } \\
\text { desviación del eje del } \\
\text { miembro. Pérdida de } \\
\text { la capacidad de vuelo. }\end{array}$ \\
\hline
\end{tabular}


Cuadro 2. Hallazgos radiográficos en nueve psitácidos rescatados de tráfico ilegal de especies silvestres y mantenidos en cautiverio en un centro de rescate en Tingo María, Huánuco, Perú (2016-2018) - Parte II

\begin{tabular}{|c|c|c|c|}
\hline $\begin{array}{l}\text { Especie / } \\
\text { extremidad } \\
\text { afectada }\end{array}$ & $\begin{array}{l}\text { Huesos / } \\
\text { articulación } \\
\text { afectada }\end{array}$ & Descripción de la lesión & Secuela clínica \\
\hline $\begin{array}{l}\text { Ara ararauna } \\
3\end{array}$ & $\begin{array}{l}\text { Huesos: } \\
\text { tibiotarso }\end{array}$ & \multirow{2}{*}{$\begin{array}{l}\text { Fractura simple en la diáfisis del } \\
\text { tibiotarso. Se observa mala } \\
\text { consolidación, formación de callo, } \\
\text { osteomielitis y artrosis de articulación } \\
\text { femoro tibiotarsiana. }\end{array}$} & \multirow{2}{*}{$\begin{array}{l}\text { Deformidad, } \\
\text { desviación del eje y } \\
\text { acortamiento del } \\
\text { miembro. Pérdida de } \\
\text { la movilidad articular } \\
\text { y claudicación } \\
\text { marcada. }\end{array}$} \\
\hline $\begin{array}{l}\text { / posterior } \\
\text { derecha }\end{array}$ & $\begin{array}{l}\text { Articulación: } \\
\text { rodilla }\end{array}$ & & \\
\hline $\begin{array}{l}\text { Ara macao } \\
\text { / anterior }\end{array}$ & $\begin{array}{l}\text { Huesos: radio } \\
\text { y ulna } \\
\text { Articulacione } \\
\text { s: codo y } \\
\text { muñeca }\end{array}$ & $\begin{array}{l}\text { Fracturas simples en diáfisis de radio } \\
\text { y ulna, ambas con desplazamiento, } \\
\text { mala consolidación, formación de } \\
\text { callo exuberante, osteomielitis y } \\
\text { artrosis de articulaciones cubital y } \\
\text { radio carpiana. }\end{array}$ & $\begin{array}{l}\text { Acortamiento del } \\
\text { miembro, desviación } \\
\text { del eje. Pérdida de } \\
\text { movimientos } \\
\text { articulares y capacidad } \\
\text { de extensión del } \\
\text { miembro y de vuelo. }\end{array}$ \\
\hline $\begin{array}{l}\text { Pionus } \\
\text { menstruus } \\
\text { / anterior } \\
\text { derecha }\end{array}$ & $\begin{array}{l}\text { Hueso: } \\
\text { húmero }\end{array}$ & $\begin{array}{l}\text { actura reciente, simple, oblicua y } \\
\text { esplazada. Se observa } \\
\text { dondeamiento de los fragmentos } \\
\text { eos fracturados e inflamación del } \\
\text { jido blando. }\end{array}$ & $\begin{array}{l}\text { Lesión sin } \\
\text { consolidación. Pérdida } \\
\text { de la capacidad de } \\
\text { vuelo. }\end{array}$ \\
\hline $\begin{array}{l}\text { Amazona } \\
\text { farinosa } \\
\text { / posterior } \\
\text { derecha }\end{array}$ & $\begin{array}{l}\text { Huesos: } \\
\text { fémur } \\
\text { Articulación: } \\
\text { rodilla }\end{array}$ & $\begin{array}{l}\text { Fractura diafisaria transversal de } \\
\text { fémur con desplazamiento lateral. Se } \\
\text { observa mala consolidación con } \\
\text { presencia de callo exuberante, } \\
\text { osteomielitis y artrosis de la } \\
\text { articulación femoro tibiotarsana }\end{array}$ & $\begin{array}{l}\text { Acortamiento del } \\
\text { miembro, desviación } \\
\text { del eje del miembro. } \\
\text { Pérdida de la } \\
\text { movilidad articular y } \\
\text { claudicación marcada. }\end{array}$ \\
\hline
\end{tabular}

transportadas en cajas de maderas o jaulas, en donde se encuentran hacinadas y propensas de sufrir traumas, ya sea por los movimientos bruscos y caídas durante el transporte, el intento de huida de ellas, o agresión entre ellas (Farrow, 2008).

La mayor frecuencia de lesiones fue en las alas (77\%), lo cual concuerda con Farrow (2008), quién menciona que el ala es la parte más susceptible a las lesiones en aves silvestres. Al respecto, McRee et al. (2017) indican que las características de conformación de los huesos largos de las aves hacen que sean más duros, pero a la vez más frágiles y menos elásticos que los huesos de los mamíferos, de allí que sean más susceptibles a sufrir fracturas. Además, la poca masa muscular y piel delgada que cubre los huesos del ala (Doneley, 2016) limita la protección del tejido óseo. 
Cuadro 3. Región anatómica y articulaciones afectadas en nueve psitácidos rescatados de tráfico ilegal de especies silvestres y mantenidos en cautiverio en un centro de rescate en Tingo María, Huánuco, Perú (20162018)

\begin{tabular}{lcccc}
\hline Extremidad & \multicolumn{2}{c}{ Región } & \multicolumn{2}{c}{ Articulación } \\
\cline { 2 - 5 } & $\mathrm{n}$ & $\%$ & $\mathrm{n}$ & $\%$ \\
\hline Ala distal & 4 & 44.4 & 3 & 42.9 \\
Ala proximal & 3 & 33.3 & 2 & 28.6 \\
Pata distal & 1 & 11.1 & 2 & 28.6 \\
Pata proximal & 1 & 11.1 & 0 & 0.0 \\
\hline Total & 9 & 100 & 7 & 100 \\
\hline
\end{tabular}

Cuadro 4. Complicaciones de lesiones en nueve psitácidos rescatados de tráfico ilegal de especies silvestres y mantenidos en cautiverio en un centro de rescate en Tingo María, Huánuco, Perú (2016-2018)

\begin{tabular}{lcc}
\hline Complicaciones & $\mathrm{n}$ & $\%$ \\
\hline Callo exuberante & 8 & 28.6 \\
Mala consolidación & 7 & 25.0 \\
Osteomielitis & 6 & 21.4 \\
Artrosis secundaria & 5 & 17.9 \\
Sin consolidación & 1 & 3.6 \\
Inflamación del tejido blando & 1 & 3.6 \\
\hline Total & 28 & 100 \\
\hline
\end{tabular}

Cuadro 5. Secuelas clínicas de las lesiones en nueve psitácidos rescatados de tráfíco ilegal de especies silvestres y mantenidos en cautiverio en un centro de rescate en Tingo María, Huánuco, Perú (2016-2018)

\begin{tabular}{lcc}
\hline Secuelas clínicas & $\mathrm{n}$ & $\%$ \\
\hline Pérdida de la capacidad de vuelo & 7 & 28.0 \\
Acortamiento del miembro afectado & 6 & 24.0 \\
Pérdida de la movilidad articular & 6 & 24.0 \\
Desviación del eje del miembro afectado & 4 & 16.0 \\
Claudicación/ dificultad para movilizarse & 2 & 8.0 \\
\hline Total & 25 & 100 \\
\hline
\end{tabular}



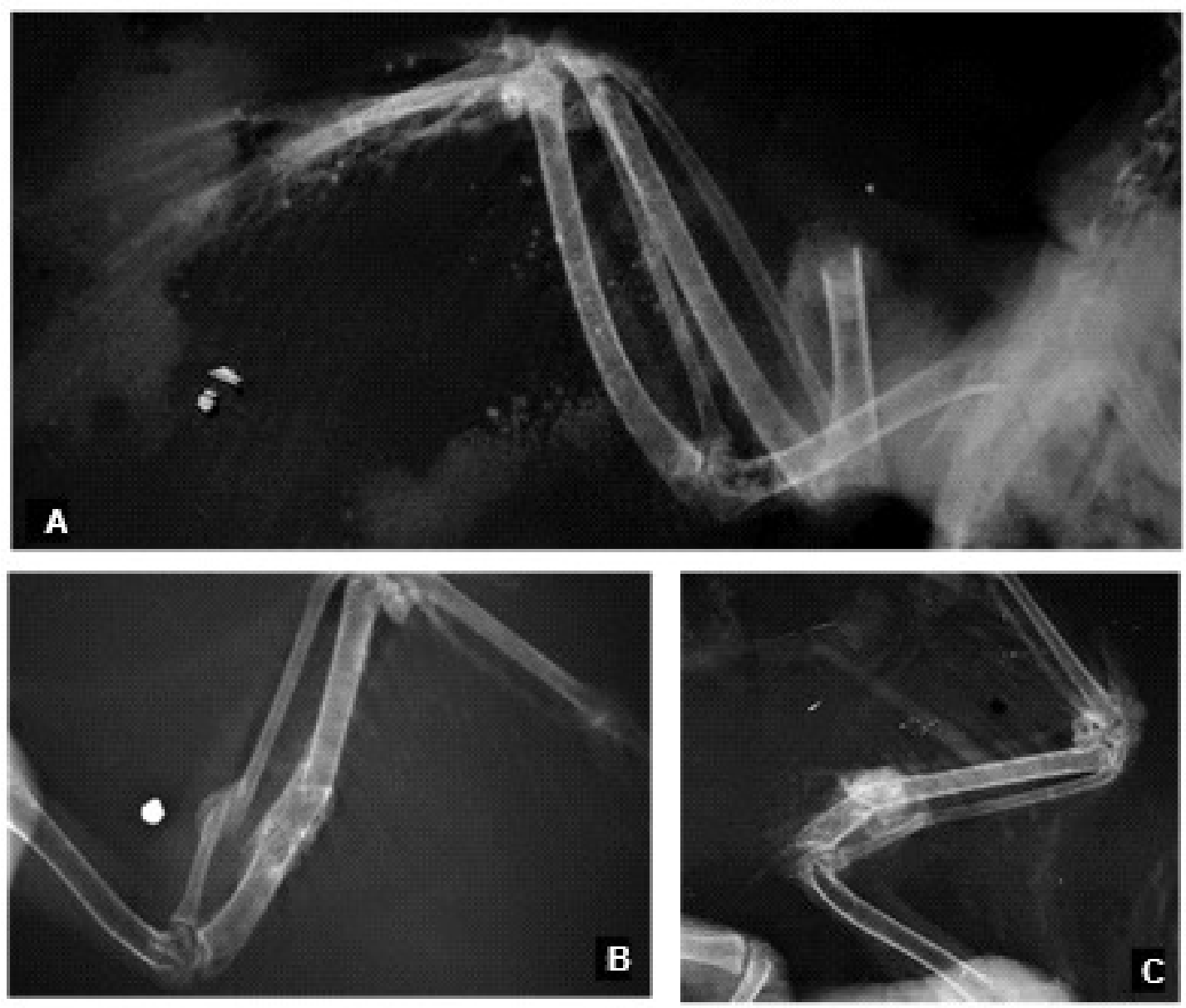

Figura 1. Vistas radiográficas medio-lateral del ala distal de aves psitácidas. A. Fractura simple, oblicua y desplazada de húmero derecho de Pionus menstruus; B. Fracturas simples y completas del radio y ulna del ala izquierda con mala consolidación. Se observa estructura radiopaca en tejido blando correspondiente a perdigón metálico de Ara militaris; C. Fractura diafisiaria de cúbito y radio cabalgada con mala consolidación de Ara ararauna.

La porción distal del ala fue la más afectada (44\%), comprometiendo tanto al radio como la ulna, sin que exista un patrón particular para las fracturas radiales y cubitales (Farrow, 2008); asimismo, se presentaron muchas fracturas desplazadas, que según algunos autores ocurren con mayor frecuencia que las no desplazadas (Farrow, 2008; Doneley, 2016).

Las complicaciones observadas en el estudio pueden ser el resultado de retrasos en la recepción del ave después de la lesión e incluir la contaminación, el compromiso del suministro vascular, las lesiones en músculo, ligamentos o tendones, o la combinación de estos factores (Redig y Cruz, 2010). Tanto el callo óseo exuberante como la mala consolidación se presentaron en todas las lesiones. Según Jones y Redig (2001), la presencia de callo exuberante es un hallazgo consistente en fracturas aviares no tratadas y se producen como resultado directo de la inestabilidad en el sitio de la fractura; asimismo los músculos de vuelo pectorales grandes tienden a distender los extremos óseos del húmero después de una 

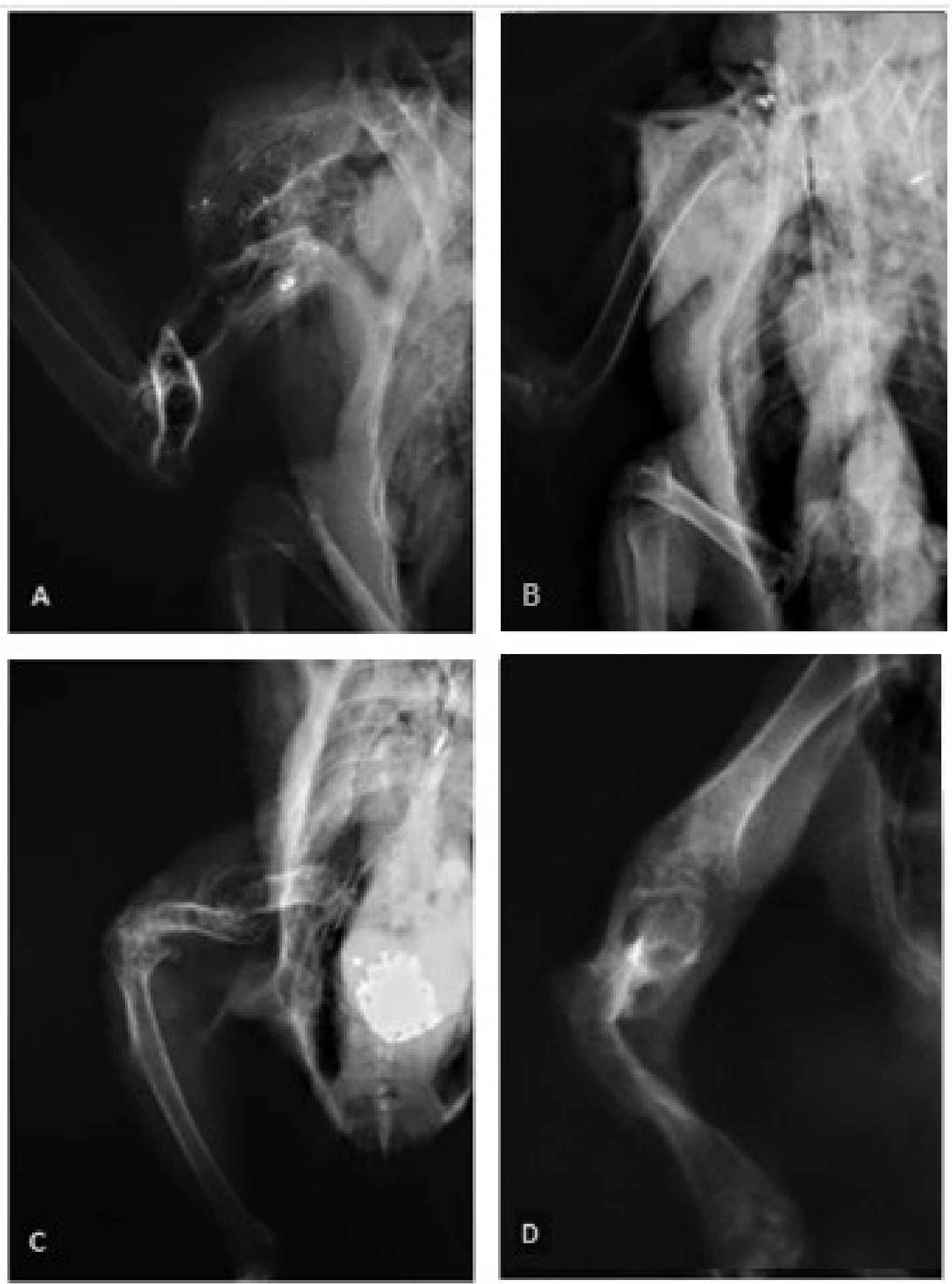

Figura 2. Vistas radiográficas medio-lateral del ala y pata de aves Psitácidas. A. Fractura y oseteomileitis del húmero (involucrando cabeza, tuberosidades y cuerpo) y la articulación humeral de Ara militaris; B. Presencia de osteomielitis con osteólisis del hueso coracoides derecho de Ara ararauna; C. Fractura de fémur derecho con mala consolidación de Amazona farinosa; D. Fractura simple en la diáfisis del tibiotarso derecho con mala consolidación y osteomielitis que afectó a la articulación femoro-tibiotarsiana (rodilla) de Ara ararauna 
fractura, lo que produce una mala alineación y pérdida de la función del ala.

Es importante indicar que reducciones aparentemente insignificantes de la longitud ósea, por ejemplo, después de una cirugía (5$10 \%$ ), o uniones defectuosas con reducción de pocos grados en el rango de movimiento de la articulación, resulta en un deterioro del vuelo, debido a la alteración en la mecánica de las alas y la interrupción del perfil aerodinámico del ala (Jones y Redig, 2001) de modo que el ave nunca podrá recuperar su nivel anterior de vuelo. Esto explica que las aves del estudio no puedan ser reintroducidas a la vida silvestre al presentar lesiones a nivel de las alas que han originado pérdida de la capacidad de vuelo. Algo similar sucedió con aquellas aves cuya lesión ocurrió a nivel de las patas, ya que los psitácidos usan los miembros pélvicos en su alimentación (Kavanagh, 1997).

\section{Literatura Citada}

1. Arnaut LS. 2006. Estudo radiográfico das afecções do sistema esquelético em aves. Tese de Mestrado. Sao Paulo, Brasil: Univ. de São Paulo. 121 p.

2. Bennett RA. 1997. Orthopedic surgery. In: Altman RB, Clubb SL, Dorrestein GM, Quesenberry K (eds). Avian medicine and surgery. Philadelphia: WB Saunders. p 733-766.

3. Beregi A, Felkai F, Molnár V, Szentgáli Z, Bíró F. 1999. Diagnostic radiology of pet and wild birds: reviewII. Indications of radiological examination and radiographs of pathological lesions. Acta Vet Hung 47: 291-302. doi: 10.1556/ AVet.47.1999.4.1

4. Cano FG 2010. Anatomía específica de aves: aspectos funcionales y clínicos. Universidad de Murcia. 17 p. [Internet]. Disponible: https://www.um.es/anatvetinteractivo/interactividad/aaves/ anatomia-aves-10.pdf
5. Chin DD, Matloff LY, Stowers AK, Tucci ER, Lentink D. 2017. Inspiration for wing design: how forelimb specialization enables active flight in modern vertebrates. J R Soc Interface: 14 20170240. doi: 10.1098/rsif.2017.0240

6. Doneley B. 2016. Avian medicine and surgery in practice: companion and aviary birds. $2^{\text {nd }}$ ed. New York, USA: CRC Press-New York. 495 p.

7. Farrow CS. 2008. Veterinary diagnostic imaging: birds, exotic pets, and wildlife. St. Louis, Missouri: Mosby Elsevier. 448p.

8. Hawkins M, Barron H, Speer B, Pollock C, Carpenter J. 2013. Exotic animal formulary. $4^{\text {th }}$ ed. St. Louis, Missouri: Elsevier Saunders. 801 p.

9. Jones R, Redig PT. 2001. Autogenous callus for repair of a humeral cortical defect in a red-tailed hawk (Buteo jamaicensis). J Avian Med Surg 15: 302309. doi: 10.1647/1082-6742(2001)015[0302:ACFROA]2.0.CO;2

10. Kavanagh M. 1997. Tibiotarsal fracture repair in a scarlet macaw using external skeletal fixation. J Small Anim Practice 38: 296-298. doi: 10.1111/j.17485827.1997.tb03469.x

11. Krautwald-Junghanns ME, Schmidt V. 2012. Aves: diagnósticos especiales, hallazgos patológicos. Cap 5: Sistema esquelético. En: Krautwald-Junghanns ME, Pees M, Reese S, Tully T (eds). Diagnóstico por imagen en animales exóticos. Barcelona, España: Multimedica Ediciones Veterinarias. p 67-78.

12. Kilbourne BM. 2013. On birds: scale effects in the neognath hindlimb and differences in the gross morphology of wings and hindlimbs. Biol J Linnean Soc 110: 14-31. doi: https://doi.org/ 10.1111/ bij. 12110

13. McRee AE, Tully TN, Nevarez JG, Sumner J, da Cunha AF. 2017. A novel surgical approach to avian femorotibiotarsal luxation repair. J Avian Med Surg 31: 156-164. doi: 10.1647/2015-139 
14. Redig PT, Cruz L. 2010. Consideraciones médicas relacionadas a traumatismos, fracturas. En: Medicina aviaria. $2^{\circ}$ ed. España: Elsevier. p 215-249.

15. SERFOR. 2017. Estrategia Nacional para reducir el tráfico ilegal de fauna silvestre en el Perú 2017-2027 y su plan de acción 2017-2022. Lima, Perú: SERFOR. $69 \mathrm{p}$.

16. Silverman S, Tell LA. 2010. Radiology of birds: an atlas of normal anatomy and positioning. St. Louis, Missouri: Saunders/ Elsevier. 299 p.
17. South N, Wyatt T. 2011. Comparing illicit trades in wildlife and drugs: an exploratory study. Deviant Behav 32: 538-561. doi: 10.1080/01639625.2010.483162

18. [WCS] Wildlife Conservation Society, [SERFOR] Servicio Nacional Forestal y de Fauna Silvestre. 2016. Guía: Manejo de animales silvestres decomisados o hallados en abandono. $2^{\circ}$ ed. Lima, Perú. 103 p. 